\title{
The impact of frailty on noninvasive mechanical ventilation in elderly medical intensive care unit patients
}

\author{
Iskender Kara $^{1} \cdot$ Muge Aydogdu $^{1} \cdot$ Gul Gursel $^{1}$
}

Received: 27 July 2017 / Accepted: 2 August 2017 / Published online: 14 August 2017

(C) Springer International Publishing AG 2017

\section{Dear Editor,}

We would like to thank Dr. Vargas and Dr. Esquinas for their interest in our study and for encouraging debate on this important and new subject the association of noninvasive ventilation (NIV) success and frailty.

As far as we understood, they recommend more clarification about the impact of comorbidities and being elderly on NIV outcome. As they also stated the number of comorbidities increases with elderly and these comorbidities along with frailty contribute to poor outcome in elderly patients even in patients treated with NIV. Since our purpose in this study is not to show the efficacy of NIV in elderly patients with chronic obstructive pulmonary disease (COPD) exacerbation or acute cardiopulmonary edema (ACPE) but to show whether frailty has any impact on NIV success in elderly patients, we did not design our study to investigate these factors. Comorbidities such as heart failure, COPD and obstructive sleep apnea are well-known disorders that are proven to be treated successfully with NIV [1,2]. Besides it is possible to find several studies investigating the usage of NIV in elderly population. Özsancak et al. evaluated patients with different age groups and reported higher NIV utilization rates among patients aged $\geq 65$ years [3]. Similarly, Schortgen et al. stated a higher rate of NIV use among elderly patients ( $>80$ years old) when compared with the younger ones ( 60 vs. $32 \%, p<0.005$ ) [4]. As being one of the factors affecting NIV success and to define our patient population we also included age, admission diagnoses and comorbidities to our tables and searched for the effect of comorbidities on NIV success, mortality and NIV application problems.

Iskender Kara

driskenderkara@gmail.com

1 Gazi Universitesi Tip Fakultesi, Ankara, Turkey
We identified no significant differences in NIV success when age and specific comorbidities were considered [5]. But since patient inclusion criteria of our study were not designed to investigate the answers of these questions we cannot interpret our results as these factors do not have any impact on NIV success. We can just say that both groups have similar percentages of patients with COPD and HF and there was no significant difference in their ages.

On the other hand and more importantly with our results, we wanted to draw attention to potential and less investigated causes of NIV failure such as Edmonton Frailty Scale (EFS) criteria (cognition, general health status, functional independence, social support, medications, nutrition, mood, and functional performance). Interestingly compared to age and comorbidities which are unmodifible factors many of EFS criteria are modifiable. We believe not only age and comorbidities but also these frailty factors should be investigated as causes of NIV failure in future.

We totally agree with these recommendations and want to thank them for their contribution.

\section{References}

1. Masip J (2008) Noninvasive ventilation in acute cardiogenic pulmonary edema. Curr Opin Crit Care 14:531-535

2. Ambrosino N, Vagheggini G (2008) Noninvasive positive pressure ventilation in the acute care setting: where are we? Eur Respir J 31:874-886

3. Ozsancak AU, Sidhom SS, Khodabandeh A et al (2016 Jan) Use and outcomes of noninvasive ventilation for acute respiratory failure in different age groups. Respir Care 61:36-43

4. Schortgen F, Follin A, Piccari L et al (2012) Results of noninvasive ventilation in very old patients. Ann Intensive Care 2:5

5. Kara I, Yildirim F, Zerman A et al (2017) The impact of frailty on noninvasive mechanical ventilation in elderly medical intensive care unit patients. Aging Clin Exp Res. doi:10.1007/s40520-017-0774-Z 